\title{
Influence of probe geometry on the response of an electrostatic probe
}

\author{
Johansson, Torben; Crichton, George C; McAllister, lain Wilson
}

Published in:

1999 Annual Report - Conference on Electrical Insulation and Dielectric Phenomena, IEEE Publication $99 \mathrm{CH} 36319$

Link to article, DOI:

10.1109/CEIDP.1999.804610

Publication date:

1999

Document Version

Publisher's PDF, also known as Version of record

Link back to DTU Orbit

Citation (APA):

Johansson, T., Crichton, G. C., \& McAllister, I. W. (1999). Influence of probe geometry on the response of an electrostatic probe. In 1999 Annual Report - Conference on Electrical Insulation and Dielectric Phenomena, IEEE Publication 99CH36319 (pp. 137-141). IEEE. https://doi.org/10.1109/CEIDP.1999.804610

\section{General rights}

Copyright and moral rights for the publications made accessible in the public portal are retained by the authors and/or other copyright owners and it is a condition of accessing publications that users recognise and abide by the legal requirements associated with these rights.

- Users may download and print one copy of any publication from the public portal for the purpose of private study or research.

- You may not further distribute the material or use it for any profit-making activity or commercial gain

- You may freely distribute the URL identifying the publication in the public portal 
1999 Conference on Electrical Insulation and Dielectric Phenomena

Influence of Probe Geometry on the Response of an

\section{Electrostatic Probe}

T. Johansson, G. C. Crichton and I. W. McAllister Department of Electric Power Engineering

Technical University of Denmark

DK-2800 Lyngby

DENMARK

\begin{abstract}
The response of an electrostatic probe is examined with reference to the probe geometry. The study involves the evaluation of the probe $\lambda$ function, from which response-related characteristic parameters can be derived. These parameters enable the probe detection sensitivity $s_{e}$ and spatial selectivity $S_{p}$ to be quantitatively assessed.
\end{abstract}

\section{Introduction}

Electrostatic probes are frequently employed to measure charges on insulating surfaces. Most probes consist essentially of a long cylindrical shaft at earth potential, with a circular conducting disc insulated from but mounted coaxially at the end of the shaft. The potential of the disc/sensor-plate is floating. Charges are electrostatically induced on the sensor plate by the ambient surface charge, and thus as the probe is moved parallel to the insulating surface the potential of the sensor plate varies. The probe sensor-plate potential, which is the measured parameter, provides the input data necessary to quantify the surface charge.

The relationship between the charge induced on the probe (change in potential) and the surface charge density at the insulating surface is expressible in terms of a general function, the $\lambda$ function [1]. Using this function, it has been possible to examine the influence of the overall system geometry upon the response of a probe [2-4]. In the present study, the influence of the probe geometry itself upon the $\lambda$ function is examined. Thereafter this knowledge enables the response of the probe with respect to detection sensitivity and spatial resolution to be quantified.

\section{The $\lambda$ Function}

The $\lambda$ function relates the charge induced on the probe to any charges within the dielectric volume or at an interface [1]. If it is assumed that the volume charge density within the solid dielectric is zero, then this relationship can be expressed simply as

$q=-\iint_{A_{0}} \lambda \sigma \mathrm{d} A$

where $q$ is the Poissonian induced charge on the sensor plate, and $\sigma$ is the surface charge density on the surface element d $A$ of $A_{\mathrm{O}}$, the surface of the solid dielectric.

The dimensionless parameter $\lambda$ is a solution of the general Laplace equation for the complete system geometry: viz.

$\vec{\nabla} \cdot(\varepsilon \vec{\nabla} \lambda)=0$

The boundary conditions are $\lambda=1$ at the probe sensor-plate and $\lambda=0$ at all other electrodes. In addition, at a dielectric interface the normal derivatives of $\lambda$ must obey the condition

$\varepsilon+\left(\frac{\partial \lambda}{\partial n}\right)_{+}=\varepsilon-\left(\frac{\partial \lambda}{\partial n}\right)_{-}$ 
where the + and - signs refer to the opposite sides of the interface. As (2) is just Laplace's equation, any standard method of solving this equation can be employed to evaluate the variation of $\lambda$ at the surface. On this occasion, solutions of Laplace's equation were obtained using a finite element software package. These solutions are then utilized to study the dependence of the probe response upon its geometry.

\section{Probe and System Geometry}

The actual geometry of the probe used in this study is shown in Fig.la, in which $r$ is the radius of the sensor plate and $x$ is the radial distance from the probe axis. The outer radius of the probe guard ring is $R$, while the gap between the sensor plate and the guard ring is $\Delta$. To minimise the influence on $\lambda$ of the probe shaft of length $L$, this latter parameter was dimensioned as large as possible. In the present case $L$ was set to be greater than $90 \mathrm{r}$. The probe itself is positioned perpendicular to a planar dielectric surface, at a height $h$ above the gas/dielectric interface; see Fig.1b.

The dielectric spacer, of relative permittivity $\varepsilon_{r}$, has a thickness $t$ in a direction parallel to the probe axis. The spacer is mounted on a grounded planar electrode, see Fig.1b. In the direction normal to the probe axis, the extent of the solid dielectric/grounded electrode is much greater than the outer diameter of the probe. With the present software this distance is set to $250 \mathrm{r}$.

\section{Results and Discussion}

With respect to the probe itself, the dimensions originally employed by Pedersen [1] were used as a reference data set: $R=6 r, \Delta=0.1 r$ and $h=0.5 r$. With respect to the spacer geometry, we select $t=20 \mathrm{r}$ and $\varepsilon_{r}=4$. The evaluation of $\lambda$ was undertaken for several values of $h, R$ and $\Delta$.

Along the dielectric interface, $\lambda$ exhibited the same bell-shaped distribution as reported previously $[1-4]$. The principal effect of varying $h, R$ and $\Delta$ was to influence the magnitude of $\lambda$, see Tables $1-3$. This affects $q$, see (1).

a)
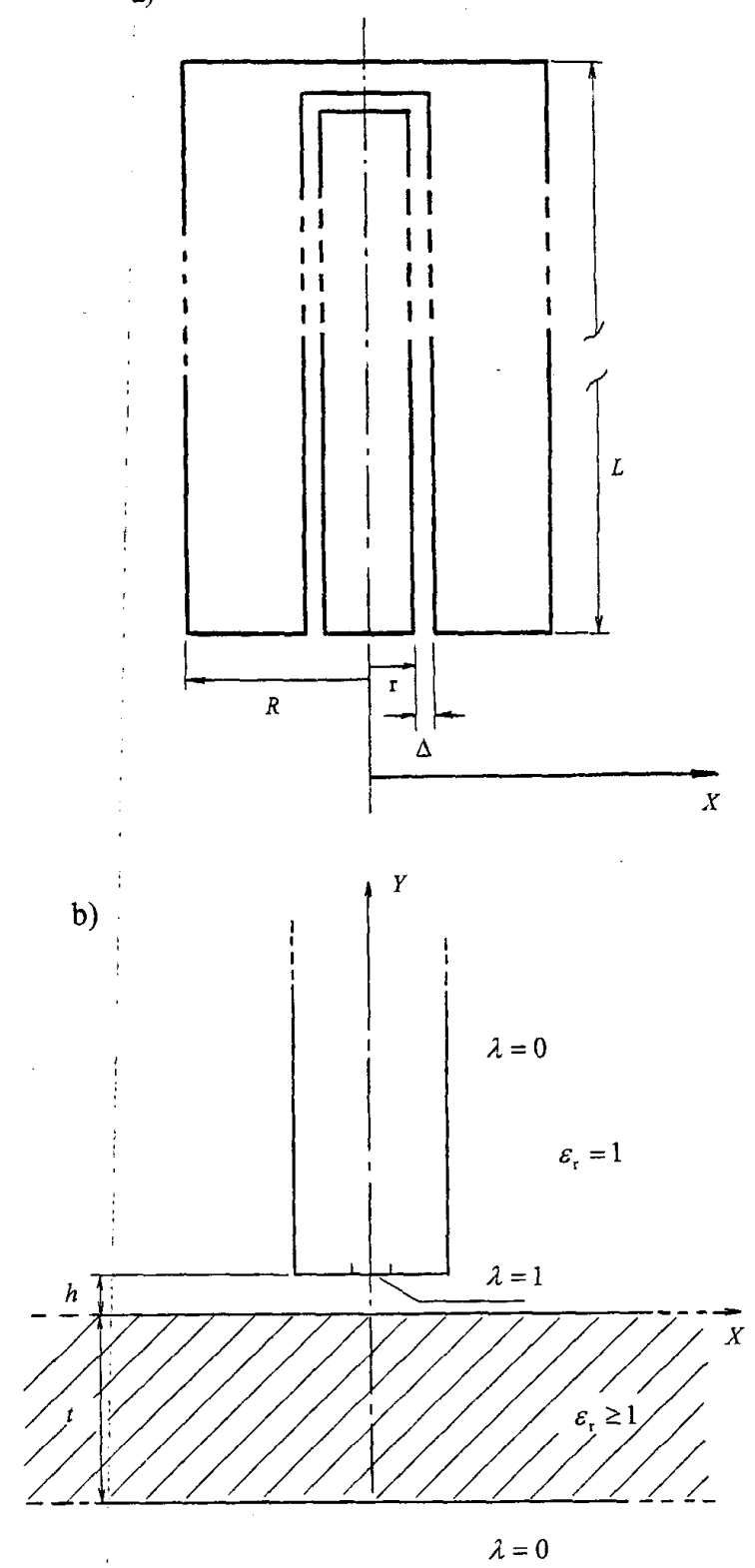

Fig.1. The probe and system geometry. 
From Table 1, with respect to the reference data set, a reduction in $h$ leads to an increase in $\lambda(0)$, a reduction in $\lambda(6 r)$ but an increase in $\lambda(60 r)$. In general, an increase in $h$ produces the opposite behaviour. Changing the value of $R$ has a negligible effect on $\lambda(0)$, see Table 2. However a reduction in the $R$ value leads to an increase in $\lambda(6 r)$ and $\lambda(60 r)$. The opposite behaviour is seen on increasing the value of $R$. This influence of $R$ is readily understood as the dimensions of the guard ring relate to the geometry of a $(\lambda=0)$ boundary. The influence of $\Delta$ upon $\lambda(0)$ is minimal, see Table 3 , although increasing values of $\Delta$ lead to an overall increase in the $\lambda(0)$ values, and vice versa. Changes in $\lambda(6 r)$ and $\lambda(60 r)$ are more marked.

The significance of the changes in the tabulated $\lambda$ values can be quantified by examining the behaviour of two other parameters; viz. $S_{\mathrm{e}}(x)$ and $S_{\mathrm{p}}(x)$. These parameters describe the detection sensitivity and the spatial resolution, respectively.

If $(x, y)$ represent cylindrical coordinates, see Fig.1b, then for a disc of constant surface density $\sigma_{0}$ located at a dielectric interface, the charge $q$ induced on the sensor plate is given by

$q(x)=-2 \pi \sigma_{0} \int_{0}^{x} \lambda\left(x^{\prime}, y\right) x^{\prime} d x^{\prime}$

where $x^{\prime}$ is a dummy variable, and a constant $y$ represents a planar interface. With respect to $q$, we introduce a detection sensitivity $s_{e}(x)$ defined as

$S_{\mathrm{e}}(x)=\left(2 / r^{2}\right) \int_{0}^{x} \lambda\left(x^{\prime}, y\right) x^{\prime} \mathrm{d} x^{\prime}$

The variation of $s_{\mathrm{e}}$ for the reference probe is indicated in Fig.2. It should be noted that $S_{\mathrm{e}}(x)=1$ when the induced charge is equal in magnitude to the
Table $1 . \lambda(x)$ data for varying $h$.

\begin{tabular}{lccc}
$h / r$ & $\lambda(0)$ & $\lambda(6 r)$ & $\lambda(60 r)$ \\
\hline 0.25 & 0.462 & $3.37(-3)$ & $3.73(-5)$ \\
0.5 & 0.289 & $4.44(-3)$ & $3.53(-5)$ \\
1.0 & 0.142 & $4.80(-3)$ & $3.04(-5)$ \\
2.0 & 0.051 & $4.29(-3)$ & $2.33(-5)$ \\
\hline
\end{tabular}

$\Delta / r=0.1, R / r=6$

Table 2. $\lambda(x)$ data for varying $R$.

\begin{tabular}{rrcc}
$R / r$ & $\lambda(0)$ & $\lambda(6 r)$ & $\lambda(60 r)$ \\
\hline 3 & 0.291 & $9.60(-3)$ & $6.20(-5)$ \\
6 & 0.289 & $4.42(-3)$ & $3.53(-5)$ \\
12 & 0.289 & $3.30(-3)$ & $1.69(-5)$ \\
\hline$\Delta / r=0.1, h / r=0.5$ &
\end{tabular}

Table 3. $\lambda(x)$ data for varying $\Delta$.

\begin{tabular}{llcc}
$\Delta / r$ & $\lambda(0)$ & $\lambda(6 r)$ & $\lambda(60 r)$ \\
\hline 0.05 & 0.283 & $4.20(-3)$ & $3.36(-5)$ \\
0.1 & 0.289 & $4.40(-3)$ & $3.52(-5)$ \\
0.2 & 0.298 & $4.80(-3)$ & $3.83(-5)$ \\
0.5 & 0.322 & $6.00(-3)$ & $4.78(-5)$ \\
\hline
\end{tabular}

$h / r=0.5, R / r=6$

Note: $(-3) \equiv 10^{-3}$

The figures in bold refer to the reference data set (reference probe).

charge directly beneath the sensor plate. From Fig.2, this equality is met for $x=9.51 r$.

With respect to the reference probe, increasing/decreasing $h$ leads to a de- 
crease/increase in $S_{\mathrm{e}}(x)$, see Fig. 2a. Reducing $R$ generates an increase in $S_{\mathrm{e}}$, especially when large areas of charge are being examined, while the opposite effect is obtained by increasing $R$, see Fig.2b. The value of $\Delta$ also influences $s_{e}$, particularly for increasing values

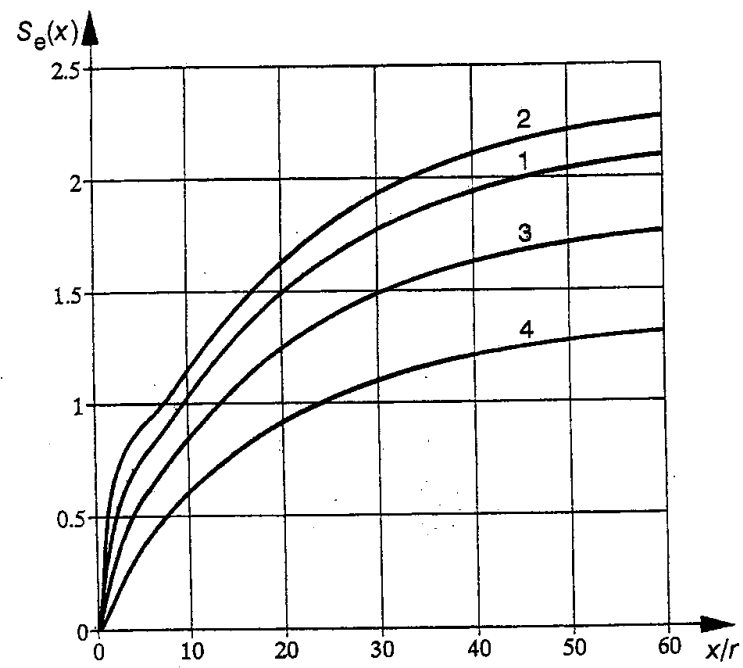

a) $1: h / r=0.5, \quad 3: h / r=1) R / r=6$ $2: h / r=0.25,4: h / r=2 \int \Delta / r=0.1$

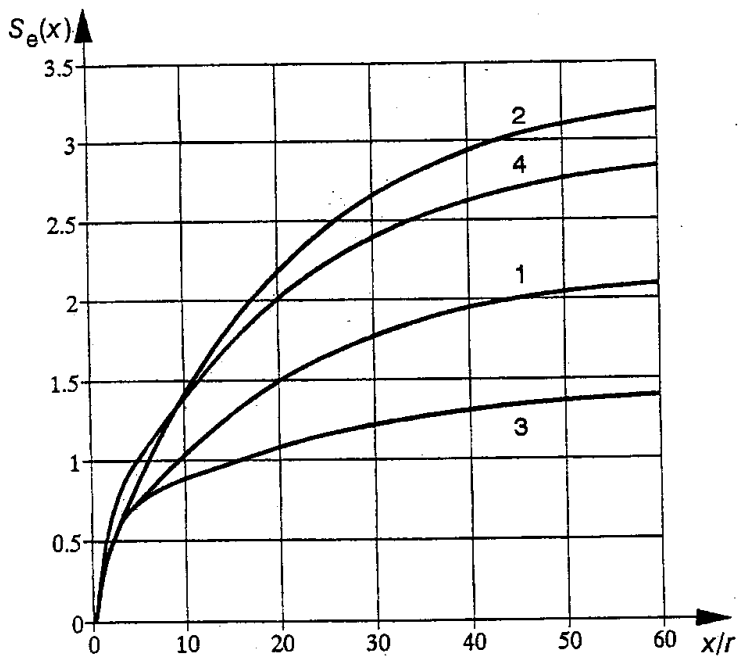

b) $1: R / r=6,2: R / r=3,3: R / r=12$ all with $h / r=0.5 \& \Delta / r 0.1$ $4: R / r=6, h / r=0.5 \& \Delta / r=0.5$

Fig. 2. Variation of $S_{e}(x)$. of $\Delta$, see Fig. 2b. Reducing $\Delta$ produces a minor reduction $(<5 \%)$.

In the context of $q$, we can also define a spatial selectivity factor $s_{p}(x)$ as follows

$s_{\mathrm{p}}(x)=\frac{\int_{0}^{x} \lambda\left(x^{\prime}, y\right) x^{\prime} \mathrm{d} x^{\prime}}{\int_{0}^{x_{0}} \lambda\left(x^{\prime}, y\right) x^{\prime} \mathrm{d} x^{\prime}}$

where $x_{0}$ is the maximum radial extent of the charged area. Again a constant charge density $\sigma_{0}$ is assumed to exist at the interface. The parameter $x_{0}$ allows the spatial selectivity of the probe to be quantified. In this study $x_{0}=60 r$.

The variation of $S_{p}$ for the reference probe is shown in Fig.3, curve 1 . For a large area of charge $\left(x_{0}=10 R\right)$, the diagram indicates that the charge not directly under the probe contributes the major portion of the induced charge.

Increasing/decreasing $h$ effectively decreases/increases $S_{p}(x)$ in the range $s_{p}(x)$

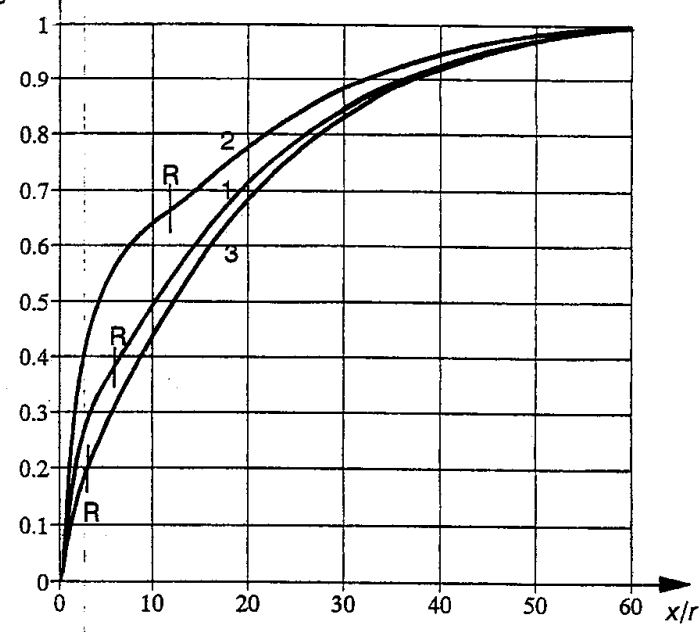

Fig.3! Variation of $S_{\mathrm{p}}(x)$. $\left.\begin{array}{l}1: R / r=6 \\ 2: R / r=12 \\ 3: R / r=3\end{array}\right\} \quad \begin{aligned} h / r=0.5 \\ \Delta / r=0.1\end{aligned}$ 
$0<x<3 R$. For the situations considered, the maximum change is $<20 \%$ at the edge of the probe. Thus although $h$ has a significant influence on $\lambda(0)$, this feature is offset by the product $\lambda(x, y) x$ in the integrals of (6).

The influence of $R$ on $S_{\mathrm{p}}$ is also shown in Fig.3. Decreasing $R$ from the reference value increases the proportion of the induced charge arising from the "external" charge. The opposite behaviour is observed by increasing $R$. Although $R$ has a negligible effect on $\lambda(0)$, the extension of the $(\lambda=0)$ boundary reduces the off-axis $\lambda$ values to such a degree that the induced charge contribution from the outer areas of charge now plays a minor rôle.

The influence of $\Delta$ on $S_{\mathrm{p}}$ is essentially negligible.

\section{Conclusion}

Through a study of a probe $\lambda$ function, the influence of the probe geometry upon the probe response was undertaken. Specifically, the study indicates the manner in which the detection sensitivity and the spatial resolution are influenced by the different geometric parameters. The guard-ring dimension exercises a dominating rôle, and can be adjusted to generate a narrow $\lambda$ distribution.

The $\lambda$ function approach provides quantitative design criteria such that for any given situation probe dimensions can be readily optimised to maximise the sensitivity. As shown in [5], a surface scanning procedure is essential. A knowledge of the probe $\lambda$ function enables a quantitative interpretation of the probe signal to be undertaken.

\section{References}

[1] A. Pedersen, "On the Electrostatics of Probe Measurements of Surface Charge Densities" in L.G. Christophorou and D.W. Bouldin, Gaseous Dielectrics $V$, New York: Pergamon Press, pp.235-240, 1987.

[2] T.O. Rerup, G.C. Crichton and I.W. McAllister, "The Response of Electrostatic Probes via the $\lambda$ Function", Conference Record of the 1994 IEEE International Symposium on Electrical Insulation, Pittsburgh, pp.82-88, 1994 .

[3] T.0. Rerup, G.C. Crichton and I.W. McAllister, "The $\lambda$ Function and the Response of Electrostatic Probes", NORD-IS 94, Nordic Insulation Symposium, Vaasa, Finland, Paper No. $7.7,1994$.

[4] T.0. Rerup, G.C. Crichton and I.W. McAllister, "Response of an Electrostatic Probe for a Right Cylindrical Spacer", 1994 Annual Report Conference on Electrical Insulation and Dielectric Phenomena, Arlington, pp.167-176, 1994.

[5] T.O. Rerup, G.C. Crichton and I.W. McAllister, "Using the $\lambda$ Function to Evaluate Probe Measurements of Charged Dielectric Surfaces", IEEE Trans. Diel. \& Elec. Insul., vol.3 pp.770-777, 1996. 